\title{
Affective symptoms in multiple system atrophy and Parkinson's disease: response to levodopa therapy
}

\author{
V Fetoni, P Soliveri, D Monza, D Testa, F Girotti
}

\begin{abstract}
The objective was to determine the extent to which psychiatric disturbances (especially mood disorders) generally considered poor prognostic factors, are present in patients with striatonigral (SND) type multiple system atrophy (MSA) compared with patients with idiopathic Parkinson's disease (IPD).

The Hamilton depression scale (HAM-D), brief psychiatric rating scale (BPRS), and Unified Parkinson's disease rating scale (UPDRS) were administered to clinically probable non-demented patients with SND-type MSA and patients with IPD matched for age and motor disability, at baseline and after receiving levodopa.

At baseline total HAM-D score was greater in patients with IPD. Overall, BPRS score did not differ between the two groups; however, patients with IPD scored higher on anxiety items of the BPRS, and patients with MSA had higher scores on the item indicating blunted affect. After levodopa, both groups improved significantly in UPDRS and HAM-D total scores (just significant for patients with MSA). Patients with IPD improved significantly in total BPRS score but patients with MSA did not.

At baseline patients with IPD were more depressed and anxious than patients with MSA who, by contrast, showed blunted affect. After levodopa, depression and anxiety of patients with IPD improved significantly whereas the affective detachment of patients with MSA did not change. Major neuronal loss in the caudate and ventral striatum, which are part of the lateral orbitofrontal and limbic circuits, may be responsible for the blunted affect not responsive to levodopa therapy found in patients with MSA.

(F Neurol Neurosurg Psychiatry 1999;66:541-544)
\end{abstract}

Keywords: parkinsonism; depression; blunted affect

Striatonigral (SND) type multiple system atrophy (MSA) is probably the most frequent form of atypical parkinsonism; in its early stages SND is difficult to distinguish from idiopathic
Parkinson's disease (IPD). However as the disease develops, autonomic, cerebellar, and pyramidal signs, as well as loss of the initial response to levodopa, ${ }^{1}$ render the differential diagnosis relatively easy. Recent studies which focused on cognitive disturbances failed to disclose clear cognitive differences between the two conditions. Both are characterised by compromise of executive functions as a result of damage to the striatofrontal circuits. ${ }^{2-4}$ However, no studies have examined psychiatric disturbances in MSA, although these have been extensively investigated in IPD. ${ }^{5-7}$ Such a study is likely to be relevant as psychiatric disturbances could have a negative impact on disease progression, as has been shown in IPD. ${ }^{8}$ The aim of this study was to determine the extent to which psychiatric disturbances, particularly mood disorders, are present in SND-type patients with MSA compared with patients with IPD with similar motor compromise.

Patients and methods

We evaluated 12 patients with probable SNDtype MSA diagnosed according to Quinn's criteria $^{9}$ and 12 patients with IPD diagnosed according to the criteria of the Parkinson's Disease Society Brain Bank. ${ }^{10}$ The patients with MSA were consecutively diagnosed at our centre for extrapyramidal diseases from December 1996 to the end of October 1997. All had orthostatic hypotension, defined as a fall in systolic blood pressure of $30 \mathrm{~mm} \mathrm{Hg}$ on standing from supine. ${ }^{11}$ The three men of the group were impotent, and all patients had disturbances of micturition without dysuria (frequency in four, urgency in five, and nocturnal incontinence in the remaining three patients). In five patients, EMG of the anal sphincter found signs of denervation. The IPD group was selected from admitted patients for similar age and overall motor state (Hoehn and Yahr scale) to the MSA group; however, duration of illness was longer in the IPD group.

None of the patients were demented according to DSM-IV criteria and all had a mini mental state examination (MMSE) score $>24$. Clinical and other characteristics of the two groups are shown in table 1 . The patients with MSA underwent cerebral MRI studies and in all cases putaminal signal hypointensity was 
Table 1 Demographic characteristics and UPDRS (part III) basal scores in the IPD and MSA patient groups

\begin{tabular}{llll}
\hline & $\begin{array}{l}\text { IPD } \\
(11 \mathrm{~F} / 1 \mathrm{M})\end{array}$ & $\begin{array}{l}\text { MSA } \\
(9 F / 3 M)\end{array}$ & p Value \\
\hline Age (y) & $58.0(6.9)$ & $58.5(6.8)$ & 0.87 \\
Illness duration (y) & $12.5(4.4)$ & $4.7(1.2)$ & 0.0001 \\
MMSE & $28.1(0.9)$ & $27.0(1.2)$ & 0.35 \\
UPDRS & $34.5(14.8)$ & $45.3(12.3)$ & 0.67 \\
\hline
\end{tabular}

present in T2 weighted images, suggesting deposition of iron or other paramagnetic substances. We used this finding to support the clinical diagnosis of SND-type MSA. ${ }^{12}$ Signs of cerebellar or brainstem atrophy on MRI were not seen in any of the patients with MSA.

All the patients with IPD were taking levodopa at a mean (SD) dosage of 1184 (758) $\mathrm{mg} /$ day and four were also receiving dopamine receptor agonists; all presented on-off phenomena and on phase hyperkinesias. The patients with MSA were also receiving levodopa at a mean (SD) dosage of 840 (348.6) mg/day.

The patients underwent baseline motor assessment at $900 \mathrm{am}-12$ hours after receiving their last levodopa dose. They were then reassessed 90 minutes after receiving $250 \mathrm{mg}$ levodopa plus $25 \mathrm{mg}$ carbidopa, when most patients said that they experienced peak benefit from the therapy.

The motor part of the unified Parkinson's disease rating scale (UPDRS) was used to assess overall motor function. Psychiatric assessment employed the Hamilton depression scale (HAM-D) $)^{13}$ supported by DSM-IV criteria for depression and the brief psychiatric rating scale (BPRS) ${ }^{14}$ to provide a general psychiatric assessment. We also calculated the scores for three HAM-D categories, formed by grouping related items as follows: category I consisting of mood depression (item 1), guilt (item 2), and suicide (item 3); category II consisting of work and interests (item 7) and retardation (item 8); category III consisting of agitation (item 9), anxiety psychic symptoms (item 10), and anxiety somatic symptoms (item 11).

We assigned the score of zero to item 14 (concerning loss of libido) of HAM-D to all patients, so that loss of libido due to autonomic involvement did not erroneously influence the depression rating. We excluded

Table 2 Comparison between scores at baseline and after levodopa on UPDRS (part III), $B P R S$, total HAM-D, and categories I, II, and III of HAM-D in IPD and MSA patient groups

\begin{tabular}{lccc}
\hline & Baseline & $\begin{array}{c}90 \text { min after } \\
\text { levodopa }\end{array}$ & $p$ Value \\
\hline Patients with IPD: & $34.5(14.8)$ & $13.2(6.2)$ & 0.0001 \\
UPDRS & $18.9(6.8)$ & $15.5(5.1)$ & 0.005 \\
BPRS & $14.9(6.8)$ & $6.8(4.9)$ & 0.002 \\
HAM-D & $3.4(2.1)$ & $1.4(2.0)$ & 0.005 \\
Category I & $1.3(1.1)$ & $0.4(0.5)$ & 0.014 \\
Category II & $3.9(2.6)$ & $1.1(1.6)$ & 0.0001 \\
Category III & & & \\
Patients with MSA: & $45.3(12.3)$ & $16.9(1.4)$ & 0.0001 \\
UPDRS & $17.1(1.1)$ & $5.1(4.3)$ & 0.19 \\
BPRS & $6.1(4.3)$ & $1.0(1.1)$ & 0.04 \\
HAM-D & $1.8(1.2)$ & $1.3(0.6)$ & 0.01 \\
Category I & $1.4(0.7)$ & $0.3(1.1)$ & 0.40 \\
Category II & $0.4(1.2)$ & 0.40 \\
Category III & & & \\
\hline
\end{tabular}

Category I (items 1, 2, 3): mood depression, guilt, suicide; category II (items 7, 8): work and interests, retardation; category III (items 9, 10, 11): agitation, anxiety psychic, anxiety somatic symptoms. items 9 and 13 (assessing depression and motor slowing) from the BPRS as these were assessed by HAM-D; for all other BPRS items a score of 3 was taken as indicating a moderate to severe symptom.

Motor assessment (with and without levodopa) was performed by one neurologist (VF) and psychiatric rating scales were administered by a second neurologist (DM). Both neurologists were experienced in the assessment of patients with movement disorders and were not blind to diagnoses for the following reasons. Firstly, the study design required that patients were assessed with and without levodopa making it easy for the examiners to guess the diagnosis in the less responsive patients. Secondly, most patients were drawn from the outpatient clinic, where they had been followed up for some years by our medical staff that included the two examiners. The data were analysed using Student's $t$ tests for paired and nonpaired data and the $\chi 2$ test for frequencies.

\section{Results}

The two groups did not differ in terms of age, motor compromise, BPRS score, or MMSE score. Duration of illness (table 1) and baseline depression score (total HAM-D) (table 2) were greater in the IPD group. Six patients with IPD had a total HAM-D score of between 18 and 29 , indicating major depression, and another six patients with IPD scored between 8 and 12, indicating dysthymia. ${ }^{15}$ Ten patients with MSA were not depressed as indicated by a total HAM-D score of $0-7$; one scored 10 (dysthymia) and one scored 18 (major depression). The baseline category I (mood depression, guilt, suicide ) and category III (agitation, and psychic and somatic anxiety) HAM-D scores were significantly higher in the IPD group than the MSA group, whereas category II (work and interests, retardation) HAM-D scores did not differ significantly (table 2 )

Ten of the 12 patients with IPD $(83.3 \%)$ had baseline scores 3 in items 1 (somatic symptoms), 2 (anxiety), and 6 (tension) of the BPRS, whereas only one patient with MSA $(8.3 \%)$ scored similarly, with a highly significant difference $(p<0.00023)$ between the groups. For item 16 (blunted affect), no patient with IPD scored 3, whereas $11 / 12$ patients with MSA $(91.7 \%)$ did so, again with a highly significant between group difference $(\mathrm{p}=0.00001)$. No patient from either group scored 3 in the remaining BPRS items.

At the second evaluation 90 minutes after levodopa administration, there was a significant improvement in the patients with IPD in motor state, and also significant changes in BPRS, total HAM-D, and HAM-D categories I, II, and III scores (table 2). In the patients with MSA there was a significant improvement in motor state, small but significant changes in HAM-D total score and category I (depression, guilt, suicide), but no changes in BPRS score, HAM-D category II, or category III scores (table 2). 


\section{Discussion}

Major depression and dysthymia are the most frequent psychiatric symptoms in IPD, with a prevalence of around $40 \%$ in hospital series and slightly lower figures in population studies. ${ }^{16-18}$ The frequency of these symptoms tends to increase as the disease progresses ${ }^{15} 19$ and there are also mood swings in relation to motor fluctuations in many patients. ${ }^{20-22}$ All our patients with IPD had advanced disease with motor fluctuations (Hoehn and Yahr stages III-IV in the off phase) as they were selected to have comparable disease severity to the patients with MSA.

Although our groups were of small size they were homogeneous (table 1); the patients with IPD were good responders to levodopa, and had long disease duration with motor fluctuations and no dementia, whereas the diagnosis of SND-type MSA was in all cases supported by MRI. We therefore consider that the groups are fairly representative of advanced stage disease populations.

Comparison of baseline assessments showed a clear difference between the groups in terms of mood and anxiety. Not surprisingly six of the fluctuating patients with IPD had a total HAM-D score >18 (major depression); by contrast only one patient with MSA had a total depression score consistent with major depression (but whose depression, anxiety, and agitation scores were quite low). Dysthymia was found in six patients with IPD and only one patient with MSA at baseline. Although we did not use a structured psychiatric interview, the diagnosis of major depression and dysthymia were supported in all cases by DSM IV criteria for depression as modified for dysthymia in neurological disorders according to the recommendations of the World Health Organisation (WHO). ${ }^{23}$

Analysis of the BPRS items showed that 11/12 patients with MSA had scores 3 for item 16 , indicating blunted affect and suggesting that these patients were emotionally indifferent to their condition - a situation that contrasts markedly with the generally anxious state of patients with IPD with regard to their compromised motor state. ${ }^{24}$

In the IPD group, anxiety and depression improved considerably at the second evaluation. According to Maricle et $a l^{25}$ at least momentary changes in depressive mood and anxiety may be a consequence of intracerebral dopamine depletion and repletion. By contrast the mood of our patients with MSA, who at baseline were not anxious or depressed as a group, was changed only slightly by levodopa. However, levodopa did bring about an improvement in the motor state of the patients with MSA, and this is consistent with reports of good motor responses to levodopa in about $30 \%$ of patients with MSA. ${ }^{26}{ }^{27}$

The emotional blunting manifested by our patients with MSA may be equivalent to the apathy and abulia known to be induced by focal lesions in the basal ganglia, particularly the caudate. ${ }^{28}$ The caudate and ventral striatum are part of the lateral orbitofrontal and limbic circuits implicated in controlling affec- tive aspects of behaviour ${ }^{29}{ }^{30}$ and major neuronal loss from the caudate is a recognised feature of the advanced stages of MSA. ${ }^{1}$ Although the MSA group as a whole was not depressed or anxious, this does not exclude the possibility that such disturbances may be present in the early stages of MSA, and as in IPD ${ }^{15} 19$ the affective state of patients with MSA may change as their disease progresses. Cognitive impairment is also likely to correlate with apathy and blunted affect. However none of our patients with MSA were demented by MMSE, although we cannot exclude the possibility that a more extensive battery of cognitive tests would show cognitive impairment. For these reasons we consider that the present study should be extended to assess affective state in relation to cognitive functions in patients with MSA in the initial phases of their illness.

\section{We thank D C Ward for help with the English.}

1 Wenning GK, Ben-Shlomo BY, Megalhães M, et al. Clinical features and natural history of multiple system atrophy: an analysis of 100 cases. Brain 1994;117:835-45.

2 Pillon B, Gouider-Khouja N, Deweer B, et al. Neuropsychological pattern of striatonigral degeneration: comparison with Parkinson's disease and progressive supranuclear palsy. F Neurol Neurosurg Psychiatry 1995;58:174-9.

3 Testa D, Fetoni V, Soliveri P, et al. Cognitive and motor performance in multiple system atrophy and Parkinson's disease compared. Neuropsychologia 1993;31:207-10.

4 Owen AM, Robbins TW. Comparative neuropsychology of parkinsonian syndromes. In: Wolters E, Scheltens P, eds. Mental dysfunction in Parkinson's disease. Proceedings of the European Congress on Mental Dysfunction in Parkinson's the European Congress on Mental Dysfunction in Parkinson's disease, Amsterdam, 20-3 Octo

5 Pillon B, Dubois B, Ploska A, et al. Severity and specificity of cognitive impairment in Alzheimer's, Huntington's and Parkinson's diseases and progressive supranuclear palsy. Neurology 1991;41:634-43.

6 Taylor AE, Saint Cyr JA, Lang AE. Frontal lobe dysfunction in Parkinson's disease. Brain 1986;109:845-83.

7 Eslinger PJ, Grattan LM. Frontal lobe and fronto-striatal substrates for different forms of human cognitive flexibility. Neuropsychologia 1993;31:17-28.

8 Starkstein SE, Preziosi TJ, Forrester AW, et al. Specificity of affective and autonomic symptoms of depression in Parkinson's disease. F Neurol Neurosurg Psychiatry 1990;53: 869-73.

9 Quinn N. Multiple system atrophy: the nature of the beast. $\mathcal{F}$ Neurol Neurosurg Psychiatry 1989;20:78-89.

10 Hughes AJ, Daniel SE, Kilford L, et al. Accuracy of clinical diagnosis of idiopathic Parkinson's disease: a clinicodiagnosis of idiopathic Parkinson's disease: a clinico-
pathological study of 100 cases. $\mathcal{f}$ Neurol Neurosurg Psychiatry 1992;55:181-4.

11 The Consensus Committee of the American Autonomic Society and the American Academy of Neurology. Consensus statement on the definition of orthostatic hypotension, pure autonomic failure, and multiple system atrophy. Neurology 1996;46:1470

12 Savoiardo M, Strada L, Girotti F, et al. MR imaging in progressive supranuclear palsy and Shy-Drager syndrome. $\mathcal{F}$ Comput Assist Tomogr 1989;13:555-60.

13 Hamilton M. A rating scale for depression. F Neurol Neurosurg Psychiatry 1960;23:56-62.

14 Overall JE, Gorham DR. The brief psychiatric rating scale. Psychol Rep 1962;10:799-812.

15 Bech P. Depressive syndrome in Parkinson's disease: clinical manifestations. In: Wolters EC, Scheltens P, eds. Proceeding manifestations. In: Wolters EC, Scheltens P, eds. Proceeding
of the European Congress on Mental Dysfunction in Parkinson's Disease, Amsterdam on 20-3 October 1993. Amsterdam: Vrije Disease, Amsterdam on 20-3

16 Tandberg E, Larsen JP, Aarsland D, et al. The occurence of depression in Parkinson's disease. Arch Neurol 1996; 53:175-9.

17 Starkstein SE, Preziosi TJ, Bolduc PL, et al. Depression in Parkinson's disease. F Nerv Ment Dis 1990; 33: 577-83.

18 Cummings JL. Depression and Parkinson's disease: a review. Am $\mathcal{F}$ Psychiatry 1992;149:443-54.

19 Huber JS, Freidenberg DL, Paulson GW, et al. The pattern of depressive symptoms varies with progression of Parkinson's disease. Feurol Neurosurg Psychiatry 1990;53:275-8. associated with the on-off phenomenon in Parkinson's disease. Psychol Med 1987;17:899-904.

21 Cantello R, Gilli M, Riccio A, et al. Mood changes associated with end of dose deterioration in Parkinson's disease: a controlled experiment. $\mathcal{f}$ Neurol Neurosurg disease: a controlled experin
Psychiatry 1986;49:1182-90. 
22 Menza MA, Sage J, Marshall E, et al. Mood changes and on-off phenomena in Parkinson's disease. Mov Disord on-off phenom $1990 ; 5: 148-51$.

23 World Health Organization. Identification of dysthymia in neurological disorders. Report on the meeting of principal investigators involved in the field trial "classification of dysthymia and related conditions in neurological disorders". Geneva: WHO, 1998; 1-14

24 Vazques A, Jimenez-Jimenez FJ, Garcia-Ruiz P, et al. "Panic attacks" in Parkinson's disease. A long term complication of levodopa therapy. Acta Neural Scand 1993;87:14-18.

25 Maricle RA, Nutt JG, Valentine RJ, et al. Dose-response relationship of levodopa with mood and anxiety in fluctuating Parkinson's disease: a double-blind placebo-controlled study. Neurology 1995;45:1757-60.
26 Hughes AJ, Colosimo C, Kleedorfer B, et al. The dopaminergic response in multiple system atrophy. $\mathcal{F}$ Neurol Neurosurg Psychiatry 1992;55:1009-13.

27 Parati EA, Fetoni V, Geminiani GC, et al. Response to L-dopa in multiple system atrophy. Clin Neuropharmacol 1993;16:139-44.

28 Bhatia KP, Mardsen CD. The behavioural and motor consequences of focal lesions of the basal ganglia in man. Brain 1994;117:859-76.

29 Cummings JL. Frontal-subcortical circuits and human behavior. Arch Neurol 1993;50:873-80.

30 Duffy JD, Coffey CE. The neurobiology of depression. In: Timble MR, Cummings JL eds. Contemporary behavioral neurology. Oxford: Butterworth-Heinemann 1997:275-89.

\section{NEUROLOGICAL PICTURE}

\section{Harlequin syndrome}

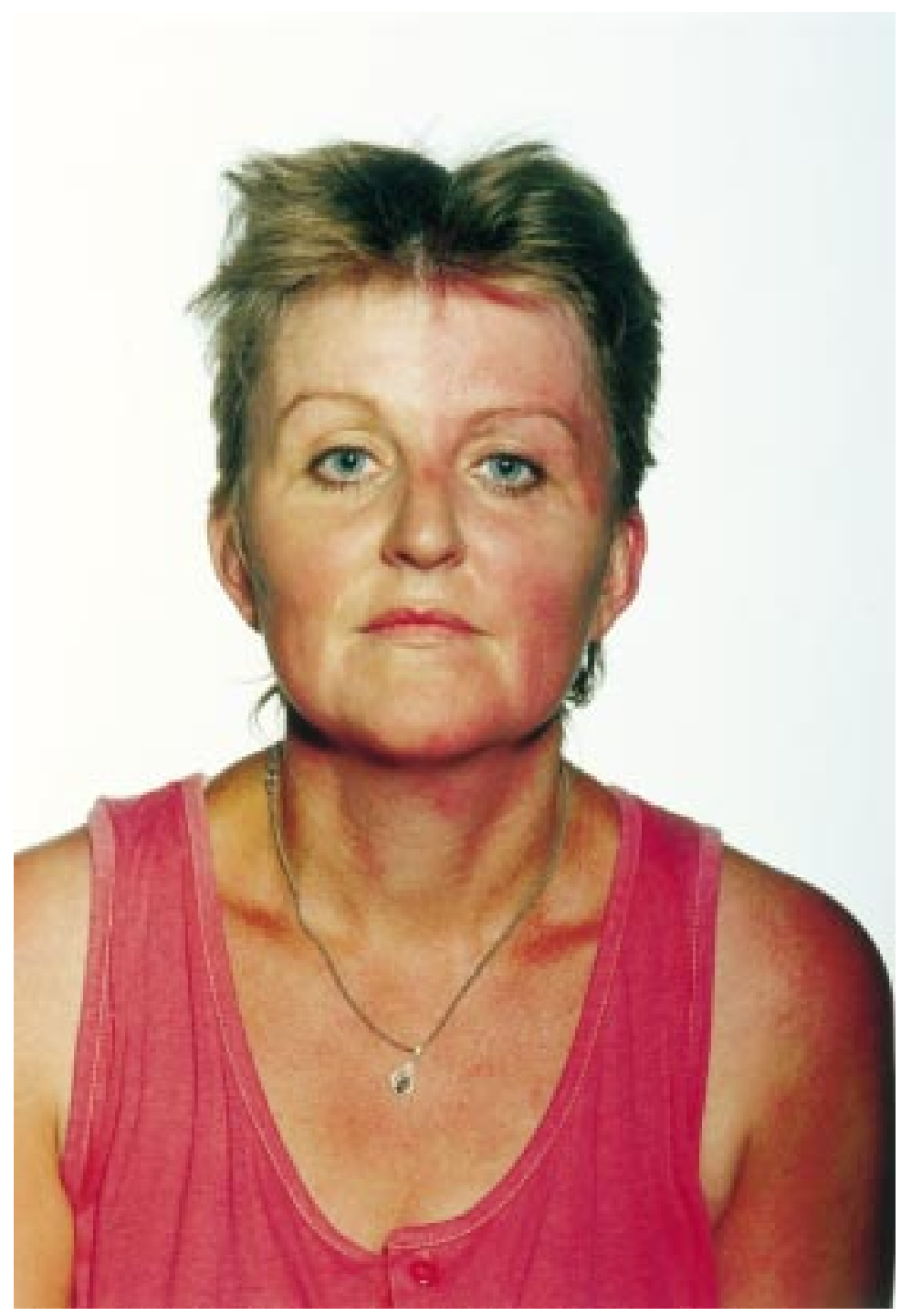

A 37 year old woman presented with a 5 month history of left hemifacial flushing induced by exercise leading to the nickname at her jogging club of half-face. She had also noticed increased warmth of the contralateral arm and sometimes the leg. Sometimes the ipsilateral eye would partially close. There was a history of migraine and ulcerative colitis. Full examination was normal apart from a subtle left ptosis. The photograph was taken after a 25 minute run.

Harlequin syndrome was first coined by Lance and Drummond in 1988 when they described five cases of unilateral flushing and sweating. ${ }^{1}$ Four of these were induced by exercise and they suggested that the lesion was due to torsional occlusion of the anterior radicular artery at the third thoracic segment. In 1993 they investigated two further patients plus two from the original report. Investigations suggested that the lesion involved both preganglionic or postganglionic cervical sympathetic fibres and parasympathetic neurons of the ciliary ganglion. ${ }^{2}$

Caparrous-Lefebvre et al described a 45 year old woman with unilateral facial loss of facial flushing and sweating with contralateral anhidrosis induced by exercise. ${ }^{3}$ She also had tonic pupils and areflexia. This was thought to be due to Ross's syndrome (Adie's syndrome with anhidrosis).

Harlequin syndrome is related to the Holmes-Adie and Ross syndromes and the persisting autonomic neuropathy of GuillainBarré syndrome.

M CORBETT

D A ABERNETHY

Department of Gastroenterology, Dunedin Hospital, 201 Great King Street, Dunedin, New Zealand

Correspondence to: Dr M Corbett, Department of Gastroenterology, Dunedin Hospital, 201 Great King Street, Dunedin, New Zealand.

1 Lance JW, Drummond P, Gandevia SC, et al. Harlequin syndrome: the sudden onset of unilateral flushing and sweating. 7NNP 1988;51:635-42.

2 Drummond P, Lance JW. Site of autonomic deficit in harlequin syndrome: local autonomic failure affecting the arm and the face. Ann Neurol 1993;34:814-19.

3 Caparrous-Lefebvre D, Hache JC, Hartevent JF, et al. Unilateral loss of facial flushing and sweating with contralateral anhidrosis: harlequin syndrome or Adie's syndrome? Clin Autonom Res 1993;3:239-41. 